\title{
Application of EFT at Thermal Energies
}

\author{
H. Sadeghi* \\ Department of Physics, University of Arak, P.O.Box 38156-879, Arak, Iran. \\ Abstract
}

We have been evaluated some observables of n-d systems by using pionless Effective Field Theory $(\operatorname{EFT}(\not t))$ and insertion of the three-body force up to next-to-next to leading order $\left(\mathrm{N}^{2} \mathrm{LO}\right)$. The evaluated data has been compared with experimental and the three-nucleon calculation of the total cross section with modern realistic two- and three-nucleon forces AV18/UrbIX potential models calculations.

*Electronic address: H-Sadeghi@araku.ac.ir 


\section{INTRODUCTION}

Formation of light nuclei from nucleons, help us for understanding of nuclear structure in Big-Bang Nucleosynthesis(BBN). For these calculations EFT is one of the most important tools. Results with this theory, in relevant range of energies, for two-body [1] and recently for three body systems [2] show very good correspondence in comparison with the experimental and evaluated results. Recently, we applied EFT( $\mathbb{E})$ to find numerical results for some astrophysical observables at thermal energies [3, 4, 5]. For these calculations all particles but nucleons are integrated out. Three-nucleon forces are added up to $\mathrm{N}^{2} \mathrm{LO}$ for cut-off independent results.

In this paper, we briefly review calculation of some BBN observables. The cross-section for neutron-deuteron capture process [4] and the photon polarization parameter $\left(R_{c}\right)$ [5] are determined. These converges order by order in low energy expansion and also is cut-off independent at this order. We have been also studied $\gamma^{3} H \rightarrow n d$ process near threshold by using $\operatorname{EFT}(\not t)[6]$. The evaluated cross section has been compared with experimental and the three-nucleon photodisintegration calculation of the total cross section with the modern realistic two- and three-nucleon forces AV18/UrbIX potential models calculations.

\section{FORMALISM}
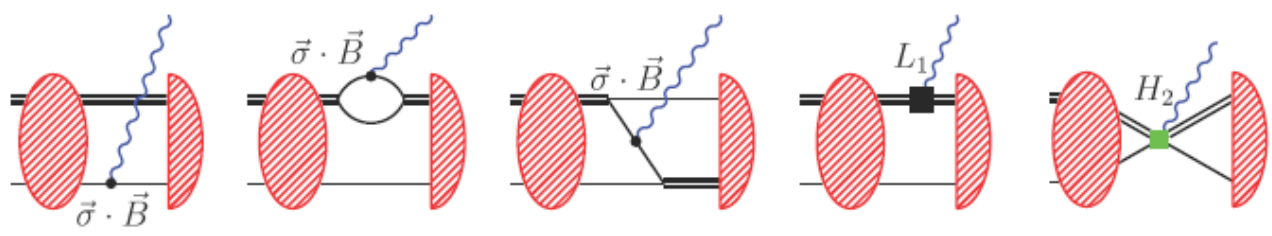

FIG. 1: The Faddeev equation for $N d$-scattering and adding photon-interaction to the Faddeev equation up to $\mathrm{N}^{2} \mathrm{LO}$. Wavy line shows photon and small circles show magnetic photon interaction. See Ref.[3,4] for notations.

The ${ }^{2} S_{1 / 2}$ describes the preferred mode for $n d \rightarrow{ }^{3} H \gamma$ and $p d \rightarrow{ }^{3} H e \gamma$ processes. The integral equation describing neutron-deuteron scattering has been discussed before [2]. The integral equation is solved numerically by imposing a cut-off $\Lambda$. In that case, a unique solution exists in the ${ }^{2} S_{1 / 2}$-channel for each $\Lambda$ and $\mathcal{H}=0$, but no unique limit as $\Lambda \rightarrow \infty$. Bedaque et al. [2] showed that the system must be stabilized by a three-body force. which absorbs all dependence on the cut-off as $\Lambda \rightarrow \infty$.

The neutron-deuteron $J=1 / 2$ phase shifts $\delta$ is determined by the on-shell amplitude $t_{t}(k, k)$, multiplied with the wave function renormalisation

$$
T(k)=Z t_{t}(k, k)=\frac{3 \pi}{M} \frac{1}{k \cot \delta-\mathrm{i} k} .
$$

For both possible magnetic dipole transitions with $J^{P}=\frac{1}{2}^{+} \quad\left(\right.$ amplitude $\left.g_{1}\right)$ and $J^{P}=\frac{3}{2}^{+}$ (amplitude $g_{3}$ ) we can write:

$$
g_{1}: \quad t^{\dagger}\left(i \vec{D} \cdot \overrightarrow{e^{*}} \times \vec{k}+\vec{\sigma} \times \vec{D} \cdot \overrightarrow{e^{*}} \times \vec{k}\right) N \quad \text { and } \quad g_{3}: \quad t^{\dagger}\left(i \vec{D} \cdot \overrightarrow{e^{*}} \times \vec{k}+\vec{\sigma} \times \vec{D} \cdot \overrightarrow{e^{*}} \times \vec{k}\right) N .
$$


and the $n d \rightarrow^{3} H \gamma$ cross section at very low energy is given by

$$
\sigma=\frac{2}{9} \frac{\alpha}{v_{r e l}} \frac{p^{3}}{4 M_{N}^{2}} \sum_{i L S J}\left[\left|\widetilde{\chi}_{i}^{L S J}\right|^{2}\right], \text { where } \tilde{\chi}_{i}^{L S J}=\frac{\sqrt{6 \pi}}{p \mu_{N}} \sqrt{4 \pi} \chi_{i}^{L S J}
$$

with $\chi$ stands for either $\mathrm{E}$ or $\mathrm{M}$ and $\mu_{N}$ is in nuclear magneton and $\mathrm{p}$ is momentum of the incident neutron in the center of mass.

\section{RESULTS AND DISCUSSION}

TABLE I: Comparison between different theoretical and experimental results for $n d \rightarrow{ }^{3} H \gamma$ and $R_{c}$. Last rows shows our EFT result up to $\mathrm{N}^{2} \mathrm{LO}$.

\begin{tabular}{|c|c|c|}
\hline Theory & $n d \rightarrow{ }^{3} H \gamma(\mathrm{mb})$ & $R_{c}$ \\
\hline $\mathrm{AV} 18 / \mathrm{IX}(\mathrm{IA}+\mathrm{MI}+\mathrm{MD}+\Delta)[7]$ & \begin{tabular}{|l|}
0.631 \\
\end{tabular} & -0.469 \\
\hline AV18/IX (gauge inv. + 3N-current) [8] & 0.578 & -0.476 \\
\hline $\operatorname{EFT}\left(\mathrm{N}^{2} \mathrm{LO}\right)[4]$ & 0.503 & -0.412 \\
\hline Experiment [9] & $0.503 \pm 0.003$ & $-0.42 \pm 0.03$ \\
\hline
\end{tabular}

We applied $\operatorname{EFT}(\not t)$ to find numerical results for some astrophysical observables in low energies. At very low energies, the interactions between nucleons can be described only by point-like interactions. All particles but nucleons are integrated out and one can identify a small, dimensionless parameter $Q=\frac{p_{\text {typ }}}{\Lambda_{\star}} \ll 1$, where $\Lambda_{\star} \sim m_{\pi}$ is the typical momentum scale at which the one pion exchange is resolved and $\operatorname{EFT}(\not \mathbb{t})$ must break down. Incident thermal neutron energies have been considered for this capture process. At these energy our calculation is dominated by only $S$-wave state and magnetic transition $M_{1}$ contribution. The $M_{1}$ amplitude is calculated up to $\mathrm{N}^{2} \mathrm{LO}$. Relevant diagrams has been shown in Fig. 1) The triton binding energy and nd scattering length in the triton channel have been used to fix them. Numerical data for these calculations can be find in the Refs. [3, 4, 5].

The cross-section for nd capture process and $R_{c}$ are in total determined as $0.503 \pm 0.003$ $\mathrm{mb}$ and $-0.412 \pm 0.003$, respectively. In table 1 , the evaluated data has been compared with experimental and the total cross section with modern realistic three-nucleon forces AV18/UrbIX potential models calculations. The cross-section for triton photodisintegration process including of $M_{1}$ and $E_{2}$ contributions at $12 \mathrm{MeV}$ is $0.866 \mathrm{mb}$ in comparison with $0.882 \mathrm{mb}$ result calculated by using AV18 and UrbIX with three-nucleon forces [10]. At the higher energies the spreads are negligible which points to a certain stability of the results and helps to identify $3 \mathrm{~N}$ force effects. Our results converges order by order in low energy expansion, is cut-off independent at this order and has a systematic error which is now smaller than the experimental error bar. 


\section{ACKNOWLEDGE}

My thanks to the $\operatorname{EFB}(20)$ organizers for his support and the warm welcome during my stay in Pisa. This work has been Supported by University of Arak.

[1] J.-W. Chen, M.J. Savage, Phys. Rev. C 60, 065205 (1999).

[2] P. F. Bedaque, H.-W. Hammer and U. van Kolck, Phys. Rev. Lett. 82, 463 (1999); Nucl. Phys. A 646, 444 (1999); Nucl. Phys. A 676, 357 (2000)..

[3] H. Sadeghi and S. Bayegan, Nucl. Phys. A 753, 291 (2005).

[4] H. Sadeghi et al., Phys. Lett. B, 643, 263 (2006).

[5] H. Sadeghi, Phys. Rev. C 75, 044002 (2007).

[6] H. Sadeghi and S. Bayegan, Submitted for publication.

[7] M. Viviani, R. Schiavilla and A. Kievsky, Phys. Rev. C 54, 534 (1996).

[8] L.E. Marcucci et al., Phys. Rev. C 72, 014001 (2005).

[9] E.T. Jurney, P.J. Bendt and J.C. Browne, Phys. Rev. C 25, 2810 (1982).

[10] R. Skibinski et al., Phys. Rev. C 67, 054001 (2003). 